\title{
Non-Steroidal Anti-Inflammatory Drug Induced Small Bowel Web Managed by Strictureplasty
}

\author{
Ahmed A. Rahman a, b, Howard S. Fan ${ }^{\mathrm{a}}$
}

\begin{abstract}
Small bowel obstructions (SBOs) are usually caused by adhesions, neoplasms, hernias or inflammatory strictures. Intramural strictures are an uncommon cause of SBO. Here we report a case of a 45-yearold female with recurrent small intestinal obstruction caused by nonsteroidal anti-inflammatory drug (NSAID) induced small bowel strictures. NSAID stricture in the small bowel was discovered by capsule endoscopy. Strictureplasty was done after presentation with small bowel obstruction.
\end{abstract}

Keywords: Small bowel obstruction; Stricture; NSAID

\section{Introduction}

Small bowel obstructions (SBOs) are usually caused by adhesions, neoplasms, hernias or inflammatory strictures. Intramural strictures are an uncommon cause of small bowel obstruction [1,2]. Here we are presenting an unusual case of sequential, stenotic stricture leading to SBO of a patient who had prior history of non-steroidal anti-inflammatory drug (NSAID) abuse.

\section{Case Report}

This patient had presented to the emergency department (ED) for four times since February, 2014 with acute onset abdominal pain and distension. On each occasion, computed tomography (CT) scan of the abdomen with intravenous and oral contrast was remarkable for SBO without any obvious cause. The patient had no history of prior abdominal surgery. She had history of NSAID-codeine abuse and hospitalisation in 2010. Brief hospitalization with narcotic analgesia and nasogastric bowel decompression was effective in resolving obstructive

Manuscript submitted November 29, 2017, accepted December 8, 2017

aDepartment of Surgery, University of New South Wales, Sutherland Hospital, Caringbah, NSW, Australia

bCorresponding Author: Ahmed A. Rahman, Department of Surgery, University of New South Wales, Sutherland Hospital, Caringbah, NSW, Australia. Email: arif_ssme@yahoo.com symptoms. The patient resumed her usual activities and diet without limitation. She had a laparoscopy for bowel obstruction before but no mechanical cause was identified. Gastric ulcer and duodenal stricture was found on endoscopy in 2013.

Outpatient capsule endoscopy indicated small bowel webs. She presented to the ED of Sutherland Hospital with abdominal pain, vomiting and obstipation. CT scan demonstrated SBO with a transition point in the right iliac fossa. She was monitored over $24 \mathrm{~h}$ of presentation, but no clinical improvement. Then she was taken to undergo a laparoscopy. Small bowel walked from ileocecal junction (ICJ) proximally until transition point identified. Small bowel was all entirely viable and pink. Transition point was marked with suture and delivered extracorporeally via entension of umbilical wound caudally. Stricture was palpable from extraluminal surface (Figs. 1 and 2). Longitudinal antimesenteric enterotomy was made centred on transition point. Fibrotic stricture in form of web was found. Enterotomy was closed in transverse manner to fashion a stricturoplasty 3-0 monocryl single layered interrupted.

Postoperatively she recovered well and was discharged once her bowels were moving.

\section{Discussion}

SBOs are most commonly caused by adhesions, hernias, neoplasms, or inflammatory strictures [1] with most caused by extraluminal adhesions due to postoperative inflammatory changes [2]. Though our patient had previous operation for SBO, she had no evidence of adhesion in her previous operations. Her primary cause of SBO was secondary to intraluminal strictures. Inflammatory bowel disease like Crohn's disease may also cause intestinal stricture. In Crohn's, obstruction

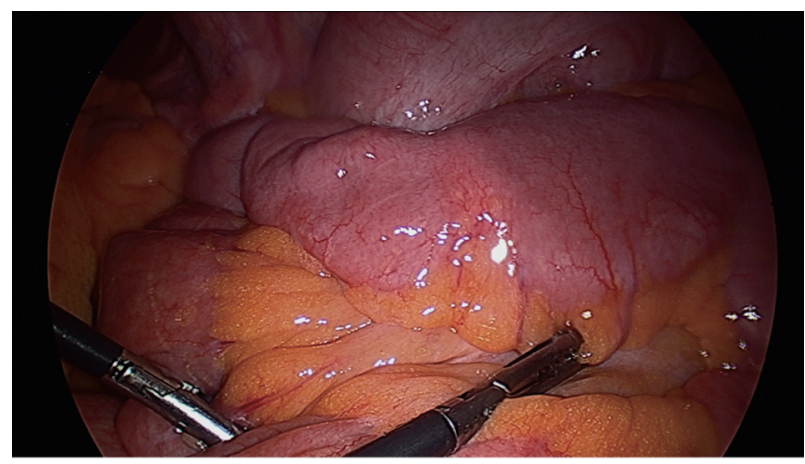

Figure 1. Transition point near stricture. 


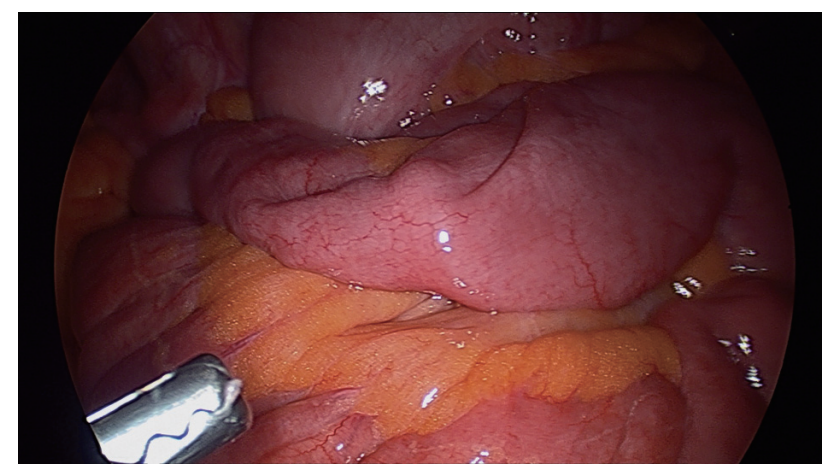

Figure 2. Small bowel web causing bowel obstruction.

may occur either due to acute inflammation or for chronic inflammation. Our patient had previous scope with no evidence of inflammatory bowel disease. Usually inflammatory bowel disease strictures are longitudinal but our patient's strictures were distinct, concentric jejunal bands [3].

Malignancy could be another cause of inflammatory strictures. Adenocarcinoma is the most common primary malignancy [4], but our patient had no history of malignancy and the post operative histopathology revealed no evidence of malignancy.

Chronic NSAIDs usage can also cause intraluminal pathology [5]. NSAIDs are one of the most commonly used medications worldwide. Although NSAIDs have great benefit, significant morbidity from chronic use of NSAIDs can affect upper gastrointestinal (UGI) tract. Most physicians are aware of NSAID complications in the UGI tract including pill-induced esophagitis and gastroduodenal ulceration and hemorrhage, but only recently has the medical community become aware of the significant potential for NSAID injury of the small intestine [6].

Small bowel enteropathy caused by NSAIDs has been hypothesized to occur through disruption of the mucosal and cellular integrity [7]. NSAIDs act as lipid-soluble weak acids that interfere with enterocyte phospholipid membranes, uncoupling of mitochondrial phosphorylation, ultimately loss of mucosal barrier and cyclooxygenase-mediated compromise of villous blood flow [8]. Diaphragm strictures are rare but pathognomonic of NSAID use. There are often multiple, 2 - 3 mm thick septa, usually in the small intestine, which can reduce the size of the intestinal lumen to a pinhole causing bowel obstruction.

\section{Conclusions}

In this case report we found sequential intraluminal stricture leading to SBO. The lack of clearly defined cause of bowel obstruction during her previous presentations with SBO made this an interesting case.

\section{References}

1. Van Buren G, 2nd, Teichgraeber DC, Ghorbani RP, Souchon EA. Sequential stenotic strictures of the small bowel leading to obstruction. World J Gastroenterol. 2007;13(40):5391-5393.

2. Markogiannakis H, Messaris E, Dardamanis D, Pararas N, Tzertzemelis D, Giannopoulos P, Larentzakis A, et al. Acute mechanical bowel obstruction: clinical presentation, etiology, management and outcome. World J Gastroenterol. 2007;13(3):432-437.

3. Hurst RD, Molinari M, Chung TP, Rubin M, Michelassi F. Prospective study of the features, indications, and surgical treatment in 513 consecutive patients affected by Crohn's disease. Surgery. 1997;122(4):661-667; discussion 667-668.

4. Gill SS, Heuman DM, Mihas AA. Small intestinal neoplasms. J Clin Gastroenterol. 2001;33(4):267-282.

5. Fortun PJ, Hawkey CJ. Nonsteroidal antiinflammatory drugs and the small intestine. Curr Opin Gastroenterol. 2005;21(2):169-175.

6. Adebayo D, Bjarnason I. Is non-steroidal anti-inflammaory drug (NSAID) enteropathy clinically more important than NSAID gastropathy? Postgrad Med J. 2006;82(965):186-191.

7. Bjarnason I, Takeuchi K, Simpson R. NSAIDs: the emperor's new dogma? Gut. 2003;52(9):1376-1378.

8. Kelly DA, Piasecki C, Anthony A, Dhillon AP, Pounder RE, Wakefield AJ. Focal reduction of villous blood flow in early indomethacin enteropathy: a dynamic vascular study in the rat. Gut. 1998;42(3):366-373. 